\title{
Zinc Complexes of New Chiral Aminophenolate Ligands: Synthesis, Characterization and Reactivity toward Lactide
}

\author{
Pascal Binda ${ }^{*}$, Kimberly Rivers ${ }^{1}$, Clifford Padgett ${ }^{2}$ \\ ${ }^{1}$ Department of Chemistry and Forensic Science, Savannah State University, Savannah, GA, USA \\ ${ }^{2}$ Department of Chemistry and Physics, Armstrong State University, Savannah, GA, USA \\ Email: "bindap@savannahstate.edu
}

Received 16 May 2016; accepted 26 July 2016; published 29 July 2016

Copyright (C) 2016 by authors and Scientific Research Publishing Inc.

This work is licensed under the Creative Commons Attribution International License (CC BY). http://creativecommons.org/licenses/by/4.0/

(c) (i) Open Access

\begin{abstract}
The syntheses of a library of new chiral aminophenolate bidentate 0,N-type ligands $\mathrm{HOC}_{6} \mathrm{H}_{4}(2-\mathrm{R}-$ 4-R') $\mathrm{CH}_{2} \mathrm{~N}(\mathrm{Me}) \mathrm{CH}(\mathrm{Me}) \mathrm{C}_{6} \mathrm{H}_{5}\left[\mathrm{R}=\mathrm{R}^{\prime}=\mathrm{Bu}^{\mathrm{t}}, \mathbf{1} ; \mathrm{R}=\mathrm{R}^{\prime}=\mathrm{Pen}^{\mathrm{t}}, 2 ; \mathrm{R}=\mathrm{Bu}^{\mathrm{t}}, \mathrm{R}^{\prime}=\mathrm{Me}, 3 ; \mathrm{R}=\mathrm{Me}, \mathrm{R}^{\prime}=\mathrm{Bu}^{\mathrm{t}}, 4 ; \mathrm{R}=\mathrm{R}^{\prime}\right.$ $=M e, 5]$ and tridentate $0, N, 0$-type ligands $H_{0 C} H_{4}\left(2,4-B u^{t}\right) C H=N C H\left(R^{\prime \prime}\right) C_{6} H_{5}\left[R^{\prime \prime}=M e, 6 ; R^{\prime \prime}=\right.$ $\mathrm{CH}_{2} \mathrm{OMe}$, 7] are reported. These ligands were characterized by elemental analysis, nuclear magnetic resonance spectroscopy $\left({ }^{1} \mathrm{H} \&{ }^{13} \mathrm{C}\right)$, and single crystal $\mathrm{X}$-ray diffraction. These ligands serve as chiral auxiliaries for inorganic chemists to design chiral metal-based complexes for asymmetric catalysis and stereoselective polymerization reactions. Three new heteroleptic zinc complexes based on these ligands have been synthesized in moderate yields via a ligand-exchange transamination reaction between homoleptic [ $\left.\mathrm{Zn}\left(\mathrm{N}\left(\mathrm{SiMe}_{3}\right)_{2}\right)_{2}\right]$ and one equivalent of corresponding ligands to afford [ $\mathrm{L}^{3} \mathrm{ZnN}$ $\left.\left(\mathrm{SiMe}_{3}\right)_{2}\right](3 \mathrm{a}),\left[\mathrm{L}^{4} \mathrm{ZnN}\left(\mathrm{SiMe}_{3}\right)_{2}\right]$ (4a), and [L7ZnN(SiMe $\left.)_{2}\right]$ (7a). Solvent-free polymerization of raclactide at $130^{\circ} \mathrm{C}$ using these zinc compounds yielded atactic polylactides with $\mathrm{M}_{\mathrm{w}} 10,000 \mathrm{~g} / \mathrm{mol}$ and narrow polydispersity of 1.3 .
\end{abstract}

\section{Keywords}

Chiral Ligands, Aminophenolate, Zinc, Lactide, Polymerization

\section{Introduction}

Due to the problems associated with conventional olefinic polymeric materials, the production of biodegradable ${ }^{*}$ Corresponding author.

How to cite this paper: Binda, P., Rivers, K. and Padgett, C. (2016) Zinc Complexes of New Chiral Aminophenolate Ligands: Synthesis, Characterization and Reactivity toward Lactide. Open Journal of Inorganic Chemistry, 6, 205-218. 
plastics from renewable resources has been a large focus in chemistry and chemical engineering research within the last two decades [1]-[16]. Aliphatic polylactides (PLAs) and polycaprolactones (PCLs) are polyesters that have been studied intensively due to their biodegradable and bioassimilable properties and have found interesting applications in packaging, drug delivery and medical implantation devices [17]-[42]. In order to obtain welldefined polyester PLA and PCL materials with predetermined microstructure, the ring-opening polymerization (ROP) of cyclic monomers (lactide and caprolactone) initiated by single-site metal complexes has been employed for chain-growth polymerization through coordination-insertion mechanism [1]-[11]. This allows for a much higher polymerization control compared to step-growth direct condensation of lactic acid.

Well-defined metal complexes of the form LMX ( $\mathrm{L}=$ multidentate ancillary ligand; $\mathrm{M}=$ central metal; $\mathrm{X}=$ initiating group) have been studied extensively to investigate the electronic and steric properties of the central metal and ancillary ligand and their effects on the polymerization process [4], [43]-[70]. Metal-based initiators of aluminum, magnesium, zinc, tin, iron, titanium, zirconium, yttrium, and lanthanide metals have been employed with reactivity increasing with metal size due to increase in electropositivity, while polymerization control decreases in the same order. As the reactivity and selectivity of a metal catalyst are largely determined by the auxiliary ligands [71]-[74], ligand design has been a central focus in ROP of cyclic esters, with aminophenolate ligands receiving great attention due to the potential to fine tune the steric and electronic properties by varying the substituent groups and pendant side-arms to afford different donor atoms, as well as their inexpensive synthetic strategies [67]-[70], [75]-[80]. Given their widespread application, it is somewhat surprising that the chiral variants of aminophenolate ligands are relatively lacking in the literature. The physical, mechanical, and thermal properties of PLA depend to a great extent on the polymer's tacticity (isotactic, syndiotactic, heterotactic and atactic). It is believed that isotactic and heterotactic PLAs produced from rac-LA will produce materials with superior properties. Thus controlling the microstructure of PLA produced from rac-LA has received great attention [6], [81]-[87] and chiral catalysts can provide a better stereo-control.

Zinc complexes are efficient catalysts for ROP of lactides with moderate reactivities compared to the highly electropositive lanthanide metals and have recently received much attention due to its flexible coordination chemistry, substitutional lability, Lewis acidity and non-toxicity [88]-[101]. With moderate reactivity of zinc allowing for more polymerization control, attaching chiral aminophenolate ancillary ligands with varying substituents and pendant arms may induce high isotactic/heterotactic selectivity in the ROP of rac-lactide. To the best of our knowledge, only Wang and Ma have reported the diastereoselective synthesis of chiral aminophenolate zinc complexes with multiple stereogenic centers and their isoselective polymerization of rac-lactide [101]. Herein we report the synthesis and characterization of new chiral aminophenolate zinc complexes with varying substituents and pendant donor arms containing one stereogenic center, and their reactivity toward racemic lactide.

\section{Experimental Procedure}

\subsection{Materials and Measurements}

All air- or moisture-sensitive reactions were carried out under a dry nitrogen atmosphere, employing standard Schlenk line and glovebox techniques. Solvents were dried over sodium/benzophenone and distilled under nitrogen. Racemic lactide was purchased from Aldrich, stored under an inert atmosphere, and used as received. Deuterated solvents were purchased from Alfa Aesar and used as received. 2,4-Di-tert-butylphenol, 2,4-dimethylphenol, 2,4-di-tert-pentylphenol, $37 \mathrm{wt} \%$ formaldehyde, and $\mathrm{D}(+)$-alpha-methylbenzylamine were purchased from Acros Organic and used as received. 3,5-Di-tert-butyl-2-hydroxybenzaladehyde was purchased from Alfa Aesar and used as received. $(R)-(+)-\mathrm{N}-\alpha$-dimethylbenzylamine, $(R)-(-)-2$-methoxy-1-phenylethylamine, and 2tert-butyl-4-methylphenol were purchased from Aldrich while 4-tert-butyl-2-methylphenol was purchased from Fluka and used as received. All ${ }^{1} \mathrm{H}$ and ${ }^{13} \mathrm{C}$ NMR spectra were recorded on a JEOL-300 NMR spectrometer and referenced to $\mathrm{CDCl}_{3}, \mathrm{C}_{6} \mathrm{D}_{6}, \mathrm{C}_{7} \mathrm{D}_{8}$, or $\mathrm{C}_{4} \mathrm{D}_{8} \mathrm{O}$. Elemental analyses (sealed ampoules under inert atmosphere for air-sensitive compounds) were performed by Midwest Microlab Incorporated in Indianapolis, IN. Melting points were obtained on a Mel-Temp apparatus and are uncorrected. Optical rotations were recorded on a Rudolph Autopol III polarimeter with sodium D-line (589 nm) at room temperature. GC-MS analyses were performed on Bruker Scion 436-GC systems at $50^{\circ} \mathrm{C}$ with electron impact ionization $(70 \mathrm{eV})$. Gel permeation chromatography (GPC) analysis was performed using Tosoh EcoSEC HLC-8320 GPC instrument and calibrated to polystyrene standards. Single crystals were analyzed at Armstrong State University in Savannah, GA using Rigaku XtaLAB 
mini X-ray diffractometer.

\subsection{Synthesis of Chiral Ligands}

\section{(R)-(+)-(2,4-Di-tert-butyl-1-hydroxylbenzyl)-N- $\alpha$-dimethylbenzylamine $\left(\mathrm{L}^{1} \mathrm{H}\right)$}

2,4-Di-tert-butylphenol (3.054 g, $14.80 \mathrm{mmol}), 37 \mathrm{wt} \%$ formaldehyde $(0.444 \mathrm{~g}, 14.80 \mathrm{mmol})$, and $(R)-(+)-\mathrm{N}-$ $\alpha$-dimethylbenzylamine $(2.000 \mathrm{~g}, 14.80 \mathrm{mmol})$ were dissolved in ethanol $(5 \mathrm{~mL})$. The resulting solution was heated at reflux for $18 \mathrm{~h}$ and then cooled to room temperature. Solvent and water were removed using high vacuum Schlenk line to obtain pale yellow oily solid. Recrystallization from ethanol at $-10^{\circ} \mathrm{C}$ (freezer) yielded an off-white solid, which was dried under high vacuum at $40^{\circ} \mathrm{C}(4.858 \mathrm{~g}, 92.8 \%)$. Mp: $73.4^{\circ} \mathrm{C}-73.6^{\circ} \mathrm{C} ;[\alpha]_{\mathrm{D}}+$ 0.301 (c $=0.04$, toluene). Elemental analysis: (Found: $\mathrm{C} 81.55, \mathrm{H} 9.94, \mathrm{~N} 4.07 . \mathrm{C}_{24} \mathrm{H}_{35} \mathrm{NO}$ requires $\mathrm{C} 81.535, \mathrm{H}$ 9.98, N 3.96. ${ }^{1} \mathrm{H}$ NMR (300 MHz; $\mathrm{CDCl}_{3}$; $\left.298 \mathrm{~K}\right) 1.29$ (s, $\left.9 \mathrm{H}, \mathrm{Ar}^{\mathrm{t}} \mathrm{Bu}\right), 1.51$ (s, $9 \mathrm{H}, \mathrm{Ar}^{\mathrm{t}} \mathrm{Bu}$ ), 1.55 (d, 3H, $J=6.87 \mathrm{~Hz}$, $\operatorname{ArCH}(\mathrm{Me}) \mathrm{NMe}$ ), 2.23 (s, 3H, $\operatorname{ArCH}(\mathrm{Me}) \mathrm{NMe}$ ), $3.77\left(\mathrm{br}, 2 \mathrm{H}, \operatorname{ArCH}_{2} \mathrm{~N}\right), 3.89(\mathrm{q}, 1 \mathrm{H}, J=6.87 \mathrm{~Hz}, \operatorname{ArCH}-$ (Me)NMe), 6.90 (s, 1H, ArH), 7.27 (s, 1H, ArH), 7.34 - 7.41 (br, 5H, ArH), 11.32 (br, 1H, ArOH). ${ }^{13} \mathrm{C}\{\mathrm{H}\}$ NMR (75 MHz; $\mathrm{CDCl}_{3}$; $\left.298 \mathrm{~K}\right) 17.3\left(\mathrm{ArCMe}_{3}\right), 29.8\left(\mathrm{ArCMe}_{3}\right), 31.9\left(\mathrm{ArCMe}_{3}\right), 34.3\left(\mathrm{ArCMe}_{3}\right), 35.0(\mathrm{ArCH}(\mathrm{Me}) \mathrm{NMe})$, $36.7(\mathrm{ArCH}(\mathrm{Me}) \mathrm{NMe})$, 59.1 ( $\mathrm{ArCH}(\mathrm{Me}) \mathrm{NMe}), 61.6\left(\mathrm{ArCH}_{2} \mathrm{~N}\right), 121.4,122.8,123.6,127.6,128.4,128.5,135.5$, 140.5, 154.7 (all $\mathrm{ArC}$ ). GC-MS m/z calcd for $\mathrm{C}_{24} \mathrm{H}_{35} \mathrm{NO}$ : 353.55; found 353.4.

\section{$(R)-(+)-(2,4-D i-t e r t-p e n t y l-1-h y d r o x y l b e n z y l)-N-\alpha$-dimethylbenzylamine $\left(\mathrm{L}^{2} \mathrm{H}\right)$}

2,4-Di-tert-pentylphenol (1.734 g, $7.40 \mathrm{mmol}), 37 \mathrm{wt} \%$ formaldehyde $(0.222 \mathrm{~g}, 7.40 \mathrm{mmol})$, and $(R)-(+)-\mathrm{N}-$ $\alpha$-dimethylbenzylamine (1.000 g, $7.40 \mathrm{mmol}$ ) were dissolved in ethanol $(5 \mathrm{~mL})$. The resulting solution was heated at reflux for $18 \mathrm{~h}$ and then cooled to room temperature. Solvent and water were removed using high vacuum Schlenk line to obtain colorless oil. Recrystallization from ethanol at $-10^{\circ} \mathrm{C}$ (freezer) yielded a white, oily solid that was dried under high vacuum at room temperature to afford a colorless oil (2.115 g, 74.9\%); $[\alpha]_{\mathrm{D}}+0.224$ (c $=0.04$, toluene). Elemental analysis: (Found: $\mathrm{C} 81.51, \mathrm{H} 10.17, \mathrm{~N} 3.78 . \mathrm{C}_{26} \mathrm{H}_{39} \mathrm{NO}$ requires C 81.84, H 10.30, N 3.67\%). ${ }^{1} \mathrm{H}$ NMR (300 MHz; $\left.\mathrm{CDCl}_{3} ; 298 \mathrm{~K}\right) 0.69$ (t, 3H $\times 2, J=7.56 \mathrm{~Hz}, \mathrm{ArCMe}_{2} \mathrm{CH}_{2} \mathrm{Me}$ ), 1.27 (s, $6 \mathrm{H}$, $\mathrm{ArCMe}_{2} \mathrm{CH}_{2} \mathrm{Me}$ ), 1.42 (s, 6H, $\mathrm{ArCMe}_{2} \mathrm{CH}_{2} \mathrm{Me}$ ), 1.52 (d, 3H, $J=6.87 \mathrm{~Hz}, \operatorname{ArCH}(M e) \mathrm{NMe}$ ), 1.61 (q, 2H, $J=7.56$ $\mathrm{Hz}, \mathrm{ArCMe}_{2} \mathrm{CH}_{2} \mathrm{Me}$ ), 1.94 (q, 2H, $J=7.56 \mathrm{~Hz}, \mathrm{ArCMe}_{2} \mathrm{CH}_{2} \mathrm{Me}$ ), 2.18 (s, 3H, $\mathrm{ArCH}(\mathrm{Me}) \mathrm{NMe}$ ), 3.73 (br, $2 \mathrm{H}$, $\mathrm{ArCH}_{2} \mathrm{~N}$ ), 3.85 (q, $1 \mathrm{H}, J=6.87 \mathrm{~Hz}, \operatorname{ArCH}(\mathrm{Me}) \mathrm{NMe}$ ), 6.76 (s, 1H, $\mathrm{ArH}$ ), 7.09 (s, 1H, ArH), 7.26 - 7.39 (br, 5H, $\mathrm{ArH}), 11.15$ (br, $1 \mathrm{H}, \mathrm{ArOH}) .{ }^{13} \mathrm{C}\{\mathrm{H}\}$ NMR (75 MHz; $\mathrm{CDCl}_{3}$; $\left.298 \mathrm{~K}\right) 9.3\left(\mathrm{ArCMe}_{2} \mathrm{CH}_{2} \mathrm{Me}\right)$, $9.7\left(\mathrm{ArCMe}_{2}-\right.$ $\left.\mathrm{CH}_{2} \mathrm{Me}\right)$, $18.7\left(\mathrm{ArCMe}_{2} \mathrm{CH}_{2} \mathrm{Me}\right), 27.7\left(\mathrm{ArCMe}_{2} \mathrm{CH}_{2} \mathrm{Me}\right)$, $28.7\left(\mathrm{ArCMe}_{2} \mathrm{CH}_{2} \mathrm{Me}\right)$, $33.0\left(\mathrm{ArCMe}_{2} \mathrm{CH}_{2} \mathrm{Me}\right)$, 36.5 ( $\mathrm{ArCMe}_{2} \mathrm{CH}_{2} \mathrm{Me}$ ), 37.3 (ArCH(Me)NMe), 38.5 ( $\left.\mathrm{ArCH}(\mathrm{Me}) \mathrm{NMe}\right), 59.1(\mathrm{ArCH}(\mathrm{Me}) \mathrm{NMe}), 61.3\left(\mathrm{ArCH}_{2} \mathrm{~N}\right)$, 121.1, 124.2, 124.9, 127.6, 128.4, 128.5, 133.7, 138.6, 154.4 (all ArC).

\section{(R)-(+)-(2-Tert-butyl-4-methyl-1-hydroxylbenzyl)-N- $\alpha$-dimethylbenzylamine $\left(\mathrm{L}^{3} \mathrm{H}\right)$}

2-Tert-butyl-4-methylphenol (2.430 g, $14.80 \mathrm{mmol}), 37 \mathrm{wt} \%$ formaldehyde $(0.444 \mathrm{~g}, 14.80 \mathrm{mmol})$, and $(R)$ (+)-N- $\alpha$-dimethylbenzylamine $(2.000 \mathrm{~g}, 14.80 \mathrm{mmol})$ were dissolved in ethanol $(5 \mathrm{~mL})$. The resulting solution was heated at reflux for $18 \mathrm{~h}$ and then cooled to room temperature. Solvent and water were removed using high vacuum Schlenk line to obtain a white solid. Recrystallization from ethanol at $-10^{\circ} \mathrm{C}$ (freezer) yielded a white powder that was dried under high vacuum at room temperature (4.010 g, 92.0\%). Yellow single crystals suitable for X-ray crystallography were grown from the supernatant solution at room temperature. Mp: $62.9^{\circ} \mathrm{C}-63.2^{\circ} \mathrm{C}$; $[\alpha]_{D}+0.232$ (c $=0.04$, toluene). Elemental analysis: (Found: $\mathrm{C}$ 81.01, $\mathrm{H} 9.33, \mathrm{~N}$ 4.55. $\mathrm{C}_{21} \mathrm{H}_{29} \mathrm{NO}$ requires $\mathrm{C}$ 80.98, H 9.385, N 4.50\%). ${ }^{1} \mathrm{H}$ NMR (300 MHz; $\mathrm{CDCl}_{3}$; $298 \mathrm{~K}$ ) 1.49 (s, 9H, $\operatorname{Ar}^{\mathrm{t}} \mathrm{Bu}$ ), 1.54 (d, 3H, $J=6.87 \mathrm{~Hz}$, $\operatorname{ArCH}(M e) \mathrm{NMe}), 2.19\left(\mathrm{~s}, 3 \mathrm{H}, \operatorname{ArCH}(\mathrm{Me}) \mathrm{NMe}\right.$ ), $2.30(\mathrm{~s}, 3 \mathrm{H}, \operatorname{ArMe}), 3.71\left(\mathrm{br}, 2 \mathrm{H}, \mathrm{ArCH}_{2} \mathrm{~N}\right), 3.89(\mathrm{q}, 1 \mathrm{H}, J=$ $6.87 \mathrm{~Hz}, \operatorname{ArCH}(\mathrm{Me}) \mathrm{NMe}), 6.70$ (s, $1 \mathrm{H}, \operatorname{Ar} H), 7.05$ (s, $1 \mathrm{H}, \operatorname{ArH}), 7.33-7.43$ (br, 5H, ArH), 11.31 (br, $1 \mathrm{H}$, ArOH). ${ }^{13} \mathrm{C}\{\mathrm{H}\}$ NMR (75 MHz; $\mathrm{CDCl}_{3}$; $\left.298 \mathrm{~K}\right)$ 17.2 $\left(\mathrm{ArCMe}_{3}\right), 20.9(\mathrm{ArMe}), 29.7\left(\mathrm{ArCMe}_{3}\right), 34.7$ (ArCH(Me)NMe), 36.5 ( $\mathrm{ArCH}(\mathrm{Me}) \mathrm{NMe}), 58.7(\mathrm{ArCH}(\mathrm{Me}) \mathrm{NMe}), 61.3\left(\mathrm{ArCH}_{2} \mathrm{~N}\right), 122.2,126.7,127.1,127.6,128.4$, 128.5, 136.3, 140.4, 154.8 (all ArC).

\section{(R)-(+)-(4-Tert-butyl-2-methyl-1-hydroxylbenzyl)-N- $\alpha$-dimethylbenzylamine $\left(\mathrm{L}^{4} \mathrm{H}\right)$}

4-Tert-butyl-2-methylphenol (1.215 g, $7.40 \mathrm{mmol}), 37 \mathrm{wt} \%$ formaldehyde $(0.222 \mathrm{~g}, 7.40 \mathrm{mmol})$, and $(R)-(+)-$ $\mathrm{N}$ - $\alpha$-dimethylbenzylamine $(1.000 \mathrm{~g}, 7.40 \mathrm{mmol})$ were dissolved in ethanol $(3 \mathrm{~mL})$. The resulting solution was heated at reflux for $18 \mathrm{~h}$ and then cooled to room temperature. Solvent and water were removed using high vacuum Schlenk line to obtain a white, oily solid. Recrystallization from ethanol was unsuccessful as only a colorless oil was obtained. Compound was purified by column chromatography (silica gel 100 mesh, hexane/ethyl acetate $=9: 1$ ) to afford a colorless oil. The oil was dissolved in hot methanol and allowed to cool to room temperature to yield a white powder, that was dried under high vacuum at $60^{\circ} \mathrm{C}(4.858 \mathrm{~g}, 92.8 \%)$. Mp: $90.4^{\circ} \mathrm{C}-$ 
90.6 $\left.6^{\circ} \mathrm{C} ; \alpha\right]_{\mathrm{D}}+0.304$ (c = 0.04, toluene). Elemental analysis: (Found: $\mathrm{C} 80.88, \mathrm{H} 9.29, \mathrm{~N} 4.61 . \mathrm{C}_{21} \mathrm{H}_{29} \mathrm{NO}$ requires C 80.98, H 9.385, N 4.50\%). ${ }^{1} \mathrm{H}$ NMR (300 MHz; $\mathrm{CDCl}_{3}$; $\left.298 \mathrm{~K}\right) 1.30$ (s, 9H, Ar ${ }^{\mathrm{t}} \mathrm{Bu}$ ), 1.56 (d, 3H, $J=$ $6.87 \mathrm{~Hz}, \operatorname{ArCH}(\mathrm{Me}) \mathrm{NMe}), 2.24$ (s, 3H, $\operatorname{ArCH}(\mathrm{Me}) \mathrm{NMe}), 2.29(\mathrm{~s}, 3 \mathrm{H}, \operatorname{ArMe}), 3.65\left(\mathrm{br}, 2 \mathrm{H}, \mathrm{ArCH}_{2} \mathrm{~N}\right), 3.82(\mathrm{q}$, $1 \mathrm{H}, J=6.87 \mathrm{~Hz}, \operatorname{ArCH}(\mathrm{Me}) \mathrm{NMe}), 6.81$ (s, $1 \mathrm{H}, \mathrm{ArH}), 7.09$ (s, 1H, ArH), 7.32 - 7.40 (br, 5H, ArH), 11.31 (br, 1H, $\mathrm{ArOH}) .{ }^{13} \mathrm{C}\{\mathrm{H}\}$ NMR (75 MHz; CDCl $\left.; 298 \mathrm{~K}\right) 16.1$ (ArMe), 17.9 ( $\left.\mathrm{ArCMe}_{3}\right), 31.7$ ( $\left.\mathrm{ArCMe}_{3}\right), 34.0$ ( $\mathrm{ArCH}-$ $(\mathrm{Me}) \mathrm{NMe}), 37.4(\mathrm{ArCH}(\mathrm{Me}) \mathrm{NMe})$, $58.6(\mathrm{ArCH}(\mathrm{Me}) \mathrm{NMe}), 63.0\left(\mathrm{ArCH}_{2} \mathrm{~N}\right), 120.7,123.0,123.7,126.7,127.7$, 128.2, 128.6, 141.1, 141.3, 153.7 (all $\operatorname{ArC}$ ).

(R)-(+)-(2,4-Di-methyl-1-hydroxylbenzyl)-N- $\alpha$-dimethylbenzylamine $\left(\mathrm{L}^{5} \mathrm{H}\right)$

2,4-Di-methylphenol (4.52 g, $37 \mathrm{mmol}), 37 \mathrm{wt} \%$ formaldehyde $(1.11 \mathrm{~g}, 37 \mathrm{mmol})$, and $(R)-(+)-\mathrm{N}-\alpha$-dimethylbenzylamine (5.00 g, $37 \mathrm{mmol})$ were dissolved in methanol $(20 \mathrm{~mL})$. The resulting solution was heated at reflux for $18 \mathrm{~h}$ and then cooled to room temperature. Solvent and water were removed using high vacuum Schlenk line to obtain a pale yellow, oily solid. Recrystallization from ethanol at $-10^{\circ} \mathrm{C}$ (freezer) yielded an off-white solid, which later became pale yellow oil upon warming to room temperature. The oil was dried under high vacuum at room temperature $(9.45 \mathrm{~g}, 94.8 \%) ;[\alpha]_{\mathrm{D}}+0.252(\mathrm{c}=0.04$, toluene). Elemental analysis: (Found: C 79.65, H 8.38, N 5.09. $\mathrm{C}_{18} \mathrm{H}_{23} \mathrm{NO}$ requires C 80.256, H 8.606, N 5.200. ${ }^{1} \mathrm{H}$ NMR (300 MHz; $\mathrm{CDCl}_{3} ; 298 \mathrm{~K}$ ) 1.59 (d, 3H, $J=6.87 \mathrm{~Hz}, \operatorname{ArCH}(M e) \mathrm{NMe}$ ), 2.23 (s, 3H, $\operatorname{ArMe}$ ), 2.29 (s, 3H, ArMe), 2.39 (s, 3H, ArCH(Me)$\mathrm{NMe}$ ), 3.70 (br, $2 \mathrm{H}, \mathrm{ArCH}_{2} \mathrm{~N}$ ), 3.87 (q, 1H, $J=6.87 \mathrm{~Hz}, \operatorname{ArCH}(\mathrm{Me}) \mathrm{NMe}$ ), $6.65(\mathrm{~s}, 1 \mathrm{H}, \mathrm{ArH}), 6.97(\mathrm{~s}, 1 \mathrm{H}, \mathrm{ArH})$, 7.38 - 7.47 (br, 5H, $\mathrm{ArH}), 11.02(\mathrm{~s}, 1 \mathrm{H}, \mathrm{ArOH}) .{ }^{13} \mathrm{C}\{\mathrm{H}\}$ NMR (75 MHz; $\left.\mathrm{CDCl}_{3} ; 298 \mathrm{~K}\right) 15.9$ (ArMe), 17.6 (ArMe), $20.6(\operatorname{ArCH}(M e) N M e), 37.3(\mathrm{ArCH}(\mathrm{Me}) \mathrm{NMe}), 58.3(\mathrm{ArCH}(\mathrm{Me}) \mathrm{NMe}), 62.9\left(\mathrm{ArCH}_{2} \mathrm{~N}\right), 114.8,121.1$, 124.6, 126.7, 127.7, 128.1, 128.7, 130.5, 140.9, 153.8 (all ArC). GC-MS m/z calcd for $\mathrm{C}_{18} \mathrm{H}_{23} \mathrm{NO}$ : 269.39; found 269.3.

\section{$(R)-(+)-\alpha$-Methyl-2-benzyl-imino-methyl-2,4-Di-tert-butyl-phenol $\left(\mathrm{L}^{6} \mathrm{H}\right)$}

3,5-Di-tert-butyl-2-hydroxybenzaldehyde (5.000 g, $21.340 \mathrm{mmol}$ ), and $\mathrm{D}(+)$-alpha-methylbenzylamine (2.586 g, $21.340 \mathrm{mmol}$ ) were dissolved in ethanol $(100 \mathrm{~mL})$. The resulting solution was heated at reflux for $18 \mathrm{~h}$ and then cooled to room temperature. Crystallization from the saturated methanol solution at room temperature yielded yellow crystals $(6.354 \mathrm{~g}, 88.2 \%)$. Mp: $93.8^{\circ} \mathrm{C}-94.1^{\circ} \mathrm{C}$; $[\alpha]_{\mathrm{D}}+0.513(\mathrm{c}=0.02$, toluene). Elemental analysis: (Found: C 81.92, H 9.16, N 4.24. $\mathrm{C}_{23} \mathrm{H}_{31} \mathrm{NO}$ requires C 81.85, H 9.26, N 4.15\%). ${ }^{1} \mathrm{H}$ NMR (500 MHz; $\mathrm{CDCl}_{3}$; $298 \mathrm{~K}) 1.34$ (s, 9H, $\left.\mathrm{Ar}^{\mathrm{t} B u}\right), 1.50\left(\mathrm{~s}, 9 \mathrm{H}, \mathrm{Ar}^{\mathrm{t}} \mathrm{Bu}\right), 1.69(\mathrm{~d}, 3 \mathrm{H}, J=6.50 \mathrm{~Hz}, \operatorname{ArCH}(\mathrm{Me}) \mathrm{N}), 4.59(\mathrm{q}, 1 \mathrm{H}, J=6.50$ $\mathrm{Hz}, \operatorname{ArCH}(\mathrm{Me}) \mathrm{N}), 7.12$ (s, 1H, ArH), $7.29(\mathrm{~s}, 1 \mathrm{H}, \mathrm{ArH}), 7.38-7.44$ (br, 5H, ArH), $8.49(\mathrm{~s}, 1 \mathrm{H}, \operatorname{ArCH}=\mathrm{N})$, 13.88 (s, $1 \mathrm{H}, \mathrm{ArOH}) .{ }^{13} \mathrm{C}\{\mathrm{H}\}$ NMR $\left(125 \mathrm{MHz} ; \mathrm{CDCl}_{3} ; 298 \mathrm{~K}\right) 24.9\left(\mathrm{ArCMe}_{3}\right), 29.5\left(\mathrm{ArCMe}_{3}\right), 31.5\left(\mathrm{ArCMe}_{3}\right)$, $34.1\left(\mathrm{ArCMe}_{3}\right), 35.1(\mathrm{ArCH}(\mathrm{Me}) \mathrm{N}), 68.5(\mathrm{ArCH}(\mathrm{Me}) \mathrm{N}), 118.0,126.0,126.5,127.2,128.6,136.7,140.1,144.1$, $158.0($ all $\mathrm{ArC}), 164.6(\mathrm{ArCH}=\mathrm{N})$.

\section{(R)-(-)- $\alpha$-Methylene- $\beta$-methoxy-2-benzyl-imino-methyl-2,4-Di-tert-butyl-phenol $\left(\mathrm{L}^{7} \mathrm{H}\right)$}

3, 5-Di-tert-butyl-2-hydroxybenzaldehyde (1.550 g, $6.613 \mathrm{mmol})$, and $(R)-(-)$-2-methoxy-1-phenylethylamine $(1.000 \mathrm{~g}, 6.613 \mathrm{mmol})$ were dissolved in ethanol $(100 \mathrm{~mL})$. The resulting solution was heated at reflux for $18 \mathrm{~h}$ and then cooled to room temperature. Solvent and water were removed using high vacuum Schlenk line to obtain a yellow solid. Recrystallization from ethanol at $-10^{\circ} \mathrm{C}$ (freezer) yielded a yellow solid (2.317 g, 95.3\%); $[\alpha]_{\mathrm{D}}-0.298$ (c $=0.02$, toluene). Yellow single crystals suitable for X-ray crystallography were grown from the supernatant solution at room temperature. Mp: $67.1^{\circ} \mathrm{C}-67.3^{\circ} \mathrm{C}$. Elemental analysis: (Found: $\mathrm{C} \mathrm{78.47,} \mathrm{H} \mathrm{8.94,} \mathrm{N}$ 4.00. $\mathrm{C}_{24} \mathrm{H}_{33} \mathrm{NO}_{2}$ requires C 78.43, $\left.\mathrm{H} 9.05, \mathrm{~N} 3.81 \%\right) .{ }^{1} \mathrm{H} \mathrm{NMR}\left(300 \mathrm{MHz} ; \mathrm{CDCl}_{3} ; 298 \mathrm{~K}\right) 1.33\left(\mathrm{~s}, 9 \mathrm{H}, \mathrm{Ar}^{\mathrm{t}} \mathrm{Bu}\right)$, 1.51 (s, 9H, $\mathrm{Ar} \mathrm{t} B u), 3.39\left(\mathrm{ArCH}(\mathrm{N}) \mathrm{CH}_{2} \mathrm{OMe}\right), 3.76\left(\mathrm{br}, 2 \mathrm{H}, \mathrm{ArCH}(\mathrm{N}) \mathrm{CH}_{2} \mathrm{OMe}\right), 4.56$ (br, $1 \mathrm{H}, \mathrm{ArCH}\left(\mathrm{N}^{2} \mathrm{CH}_{2^{-}}\right.$ OMe), 7.14 (s, $1 \mathrm{H}, \mathrm{ArH}), 7.29-7.44(\mathrm{br}, 6 \mathrm{H}, \mathrm{ArH}), 8.46(\mathrm{~s}, 1 \mathrm{H}, \mathrm{ArCH}=\mathrm{N}), 11.69(\mathrm{br}, 1 \mathrm{H}, \mathrm{ArOH}) .{ }^{13} \mathrm{C}\{\mathrm{H}\}$ NMR (75 MHz; $\left.\mathrm{CDCl}_{3} ; 298 \mathrm{~K}\right) 29.6\left(\mathrm{ArCMe}_{3}\right), 31.6\left(\mathrm{ArCMe}_{3}\right), 34.3\left(\mathrm{ArCMe}_{3}\right), 35.2\left(\mathrm{ArCMe}_{3}\right), 59.0(\mathrm{ArCH}-$ $\left.(\mathrm{N}) \mathrm{CH}_{2} \mathrm{OMe}\right), 59.4\left(\mathrm{ArCH}(\mathrm{N}) \mathrm{CH}_{2} \mathrm{OMe}\right), 73.7\left(\mathrm{ArCH}(\mathrm{N}) \mathrm{CH}_{2} \mathrm{OMe}\right), 118.1,126.5,127.3,127.8,128.8,136.7$, 140.0, 140.2, 140.6, $158.1($ all $\mathrm{ArC}), 166.8(\mathrm{ArCH}=\mathrm{N})$. GC-MS m/z calcd for $\mathrm{C}_{24} \mathrm{H}_{33} \mathrm{NO}_{2}$ : 337.51; found 337.6.

\subsection{Synthesis of Chiral Zinc Complexes}

\section{$\left[\mathrm{L}^{3} \mathrm{ZnN}\left(\mathrm{SiMe}_{3}\right)_{2}\right]$ (3a)}

To a colorless toluene solution $(10 \mathrm{~mL})$ of $\mathrm{Zn}\left[\mathrm{N}\left(\mathrm{SiMe}_{3}\right)_{2}\right]_{2}(1.545 \mathrm{~g}, 4.000 \mathrm{mmol})$ at $-20^{\circ} \mathrm{C}$, a colorless hexane solution $(20 \mathrm{~mL})$ of ligand $\mathbf{L}^{3}(1.246 \mathrm{~g}, 4.000 \mathrm{mmol})$ was added drop wise under nitrogen. The resulting reaction mixture was stirred gently $(4 \mathrm{~h})$ as it warmed to $12^{\circ} \mathrm{C}$ to afford a clear pale yellow solution of 3a. Volatiles were removed in vacuo without heating to afford a yellow foam-like oily solid that was washed with hexanes and dried under high vacuum to afford 3a $(1.15 \mathrm{~g}, 53.6 \%)$; Mp: $97.1^{\circ} \mathrm{C}-97.4^{\circ} \mathrm{C} ;[\alpha]_{\mathrm{D}}+0.056(\mathrm{c}=0.01$, 
toluene). Elemental analysis: (Found: C 60.38, H 8.61, N 5.29. $\mathrm{C}_{27} \mathrm{H}_{46} \mathrm{~N}_{2} \mathrm{OZnSi}_{2}$ requires C 60.48, H 8.65, N 5.22\%). ${ }^{1} \mathrm{H}$ NMR (300 MHz; $\mathrm{C}_{4} \mathrm{D}_{8} \mathrm{O}$; $298 \mathrm{~K}$ ) $0.02-0.008$ (br, 18H, N(SiMe $\left.)_{2}\right), 1.26$ (s, 9H, Ar ${ }^{\mathrm{B} u}$ ), 1.40 (br, 3H, ArMe), 2.02 (br, 3H, $\operatorname{ArCH}(M e) N M e), 2.16$ (br, 1H, ArCH(Me)NMe), 2.40 (s, 3H, ArCH(Me)NMe), 4.39 (br, 2H, $\mathrm{ArCH}_{2} \mathrm{~N}$ ), 6.21 (s, $\left.1 \mathrm{H}, \mathrm{ArH}\right), 6.85$ (s, 1H, $\mathrm{ArH}$ ), 7.32 - 7.46 (br, 5H, $\mathrm{ArH}$ ).

\section{$\left[\mathrm{L}^{4} \mathrm{ZnN}\left(\mathrm{SiMe}_{3}\right)_{2}\right]$ (4a)}

To a colorless toluene solution $(10 \mathrm{~mL})$ of $\mathrm{Zn}\left[\mathrm{N}\left(\mathrm{SiMe}_{3}\right)_{2}\right]_{2}(1.545 \mathrm{~g}, 4.000 \mathrm{mmol})$ at $-20^{\circ} \mathrm{C}$, a colorless hexane solution ( $20 \mathrm{~mL}$ ) of ligand $\mathbf{L}^{4}$ (1.246 g, $4.000 \mathrm{mmol}$ ) was added drop wise under nitrogen. The resulting reaction mixture was stirred gently $(4 \mathrm{~h})$ as it warmed to $12^{\circ} \mathrm{C}$ to afford a clear pale yellow solution of $4 \mathbf{a}$. Volatiles were removed in vacuo without heating to afford a pale-yellow (off white) solid which was washed twice with hexanes and dried under high vacuum to afford $4 \mathrm{a}(1.10 \mathrm{~g}, 51.3 \%)$; Mp: $119.8^{\circ} \mathrm{C}-120.1^{\circ} \mathrm{C} ;[\alpha]_{\mathrm{D}}+0.068$ (c = 0.01, toluene). Elemental analysis: (Found: C 60.40, H 8.61, N 5.07. $\mathrm{C}_{27} \mathrm{H}_{46} \mathrm{~N}_{2} \mathrm{OZnSi}$ requires C 60.48, H 8.65, N 5.22\%). ${ }^{1} \mathrm{H}$ NMR (300 MHz; $\mathrm{C}_{4} \mathrm{D}_{8} \mathrm{O}$; $298 \mathrm{~K}$ ) 0.04 (s, 18H, N(SiMe $)_{2}$ ), 1.12 (s, 9H, $\mathrm{Ar}^{\mathrm{t}} \mathrm{Bu}$ ), 1.21 (br, 3H, ArMe), 1.46 (br, 3H, $\operatorname{ArCH}(\mathrm{Me}) \mathrm{NMe}$ ), 2.20 (br, 1H, ArCH(Me)NMe), 2.29 (s, 3H, $\operatorname{ArCH}(\mathrm{Me}) \mathrm{NMe}$ ), 4.51 (br, 2H, $\mathrm{ArCH}_{2} \mathrm{~N}$ ), 6.48 (s, $1 \mathrm{H}, \mathrm{ArH}$ ), 7.00 - 7.16 (br, 5H, ArH), 7.39 (s, 1H, ArH).

\section{$\left[\mathrm{L}^{7} \mathrm{ZnN}\left(\mathrm{SiMe}_{3}\right)_{2}\right](\mathbf{7 a})$}

To a colorless THF solution $(10 \mathrm{~mL})$ of $\mathrm{Zn}\left[\mathrm{N}\left(\mathrm{SiMe}_{3}\right)_{2}\right]_{2}(1.545 \mathrm{~g}, 4.000 \mathrm{mmol})$ at $-20^{\circ} \mathrm{C}$, a clear yellow THF solution (15 mL) of ligand $\mathbf{L}^{7}(1.470 \mathrm{~g}, 4.000 \mathrm{mmol})$ was added drop wise under nitrogen. The resulting reaction mixture was stirred gently $(4 \mathrm{~h})$ as it warmed to $12^{\circ} \mathrm{C}$ to afford a clear yellow solution of $7 \mathbf{a}$ that was put in a freezer $\left(-56^{\circ} \mathrm{C}\right)$ to grow crystals. Yellow crystals of $7 \mathbf{a}$ were obtained at $-56^{\circ} \mathrm{C}$ that later dissolved at room temperature. Volatiles were removed in vacuo without heating to afford a yellow oily solid that was recrystallized from a THF/hexane mixture at $-56^{\circ} \mathrm{C}$ and dried under high vacuum to afford $7 \mathbf{a}$ (1.40 g, 53.5\%); Mp: $211.9^{\circ} \mathrm{C}-212.3^{\circ} \mathrm{C},[\alpha]_{\mathrm{D}}-0.052$ (c $=0.01$, toluene). Elemental analysis: (Found: C 62.21, H 8.49, N 4.58 . $\mathrm{C}_{34} \mathrm{H}_{48} \mathrm{~N}_{2} \mathrm{O}_{3} \mathrm{ZnSi}_{2}$ requires C 62.41, H 7.39, N 4.28\%). ${ }^{1} \mathrm{H}$ NMR (300 MHz; $\left.\mathrm{C}_{4} \mathrm{D}_{8} \mathrm{O} ; 298 \mathrm{~K}\right) 0.04$ (s, $18 \mathrm{H}$, $\left.\mathrm{N}\left(\mathrm{SiMe}_{3}\right)_{2}\right), 1.30$ (s, 9H, $\left.\mathrm{Ar}^{\mathrm{t}} \mathrm{Bu}\right), 1.45$ (s, 9H, $\left.\mathrm{Ar}^{\mathrm{t}} \mathrm{Bu}\right), 3.09\left(\mathrm{ArCH}(\mathrm{N}) \mathrm{CH}_{2} \mathrm{OMe}\right), 4.12(\mathrm{br}, 2 \mathrm{H}, \operatorname{ArCH}(\mathrm{N})-$ $\mathrm{CH}_{2} \mathrm{OMe}$ ), 4.81 (br, $\left.1 \mathrm{H}, \mathrm{ArCH}(\mathrm{N}) \mathrm{CH}_{2} \mathrm{OMe}\right), 6.84(\mathrm{~s}, 1 \mathrm{H}, \mathrm{ArH}), 6.95(\mathrm{br}, 5 \mathrm{H}, \mathrm{ArH}), 8.06(\mathrm{~s}, 1 \mathrm{H}, \mathrm{ArCH}=\mathrm{N})$.

\subsection{Polymerization Procedure}

Appropriate amount of zinc compound and racemic lactide were measured and put in an oven-dried Schlenk flask. The flask was subjected to heating at $130^{\circ} \mathrm{C}$. After appropriate reaction time, the polymerization reaction was quenched with $1 \mathrm{ml}$ of acidified methanol and the polymer was isolated and analyzed.

\section{Results and Discussion}

\subsection{Synthesis of Chiral Ancillary Ligands}

The chiral ligands $\mathbf{L}^{\mathbf{1}} \mathbf{H}-\mathbf{L}^{\mathbf{5}} \mathbf{H}$ (Figure 1) were synthesized via Mannich condensation reactions using inexpensive substituted phenols, formaldehyde and $(R)-(+)-\mathrm{N}-\alpha$-dimethylbenzylamine in refluxing ethanol (Scheme 1). Meanwhile, the chiral Schiff base ligand $\mathbf{L}^{6} \mathbf{H}$ and $\mathbf{L}^{7} \mathbf{H}$ were synthesized through condensation reactions of 3, 5-Di-tert-butyl-2-hydroxybenzaldehyde with the corresponding chiral amine, $(R)-(+)-\mathrm{N}-\alpha$-dimethylbenzylamine and $(R)-(-)-2$-methoxy-1-phenylethylamine, respectively (Scheme 2). The synthesized ligands were characterized<smiles>CCC(C)(C)c1cc(C(C)(C)C)cc(CN(C)[C@@H](C)c2ccccc2)c1O</smiles>

Figure 1. New chiral [ON] and [ONO] aminophenolate ligands. 
using NMR, elemental analysis, and GC-MS to ascertain the structures. X-ray crystallography of single crystals of ligands $\mathbf{L}^{3} \mathbf{H}$ and $\mathbf{L}^{7} \mathbf{H}$ indeed supported NMR spectra and elemental analysis.

The use of different phenolic substituents (methyl, butyl and pentyl) will provide a library of compounds suitable for metal catalytic investigations in polymerization reactions. Attachment of synthesized ligands to zinc, calcium, tin and palladium metals would offer new research opportunities in asymmetric synthesis and metal catalyzed ring-opening polymerization of lactones. There is great interest in investigating the effect of one stereogenic center in conjunction with phenolic bulky substituent on catalytic selectivity. The [ON] ligands are expected to be bidentate with the possibility of having a tridentate coordination via the phenyl pendant arms while the [ONO] ligand has an additional coordinating oxygen atom.

\subsection{Synthesis of Zinc Complexes}

Three new zinc compounds 3a, $\mathbf{4 a}$, and $\mathbf{7 a}$ have been synthesized in moderate yields, via acid-base transamination reactions employing one equivalent of the zinc precursor, $\left.\mathrm{Zn}\left(\mathrm{N}_{(\mathrm{SiMe}}\right)_{2}\right)_{2}$, and one equivalent of the ligands $\mathbf{L}^{3} \mathbf{H}, \mathbf{L}^{4} \mathbf{H}$ and $\mathbf{L}^{7} \mathbf{H}$ in toluene and THF to afford $\left[\mathrm{L}^{3} \mathrm{ZnN}\left(\mathrm{SiMe}_{3}\right)_{2}\right]$ (3a) $\left[\mathrm{L}^{4} \mathrm{ZnN}\left(\mathrm{SiMe}_{3}\right)_{2}\right]$ (4a) and $\left[\mathrm{L}^{7} \mathrm{ZnN}\left(\mathrm{SiMe}_{3}\right)_{2}\right]$ (7a) (Scheme 3). Compounds $\mathbf{3 a}$ and $\mathbf{4 a}$ were synthesized in toluene while $\mathbf{7 a}$ was synthesized in THF. The synthesized zinc compounds have been fully characterized by NMR spectroscopy, elemental analysis and melting point (see experimental). ${ }^{1} \mathrm{H}$ NMR spectroscopy of compounds $\mathbf{3 a}$ and $\mathbf{4 a}$ in $\mathrm{d}_{8}$-toluene $\left(\mathrm{C}_{7} \mathrm{D}_{8}\right)$ revealed two distinct ligand environments (in approximate equal ratio); likely caused by molecular dimerization to satisfy the coordinatively unsaturated zinc metal. The silylamide ligand was represented by two peaks of approximate equal intensity. However, NMR spectroscopy of 3a and 4a in the presence of a donor solvent such as<smiles>[R]c1cc([R])c(O)c([1H])c1</smiles><smiles>CN[C@H](C)c1ccccc1</smiles>

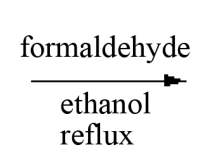

$$
\begin{aligned}
& \mathrm{L}^{1} \mathrm{H}=\mathrm{R}_{1}=\mathrm{R}_{2}={ }^{\mathrm{t}} \mathrm{Bu} \\
& \mathrm{L}^{2} \mathrm{H}=\mathrm{R}_{1}=\mathrm{R}_{2}=\mathrm{Pe} \\
& \mathrm{L}^{3} \mathrm{H}=\mathrm{R}_{1}=\mathrm{Me} ; \mathrm{R}_{2}={ }^{\mathrm{t}} \mathrm{Bu} \\
& \mathrm{L}^{4} \mathrm{H}=\mathrm{R}_{1}=\mathrm{Bu} ; \mathrm{R}_{2}=\mathrm{Me} \\
& \mathrm{L}^{5} \mathrm{H}=\mathrm{R}_{1}=\mathrm{R}_{2}=\mathrm{Me}
\end{aligned}
$$<smiles>[R]c1cc([R2])c(O)c(CN(C)[C@H](C)c2ccccc2)c1</smiles>

$\mathrm{L}^{1-5} \mathrm{H}$

Scheme 1. Synthesis of ligands $\mathrm{L}^{1} \mathrm{H}-\mathrm{L}^{5} \mathrm{H}$ via Mannich condensation reactions.

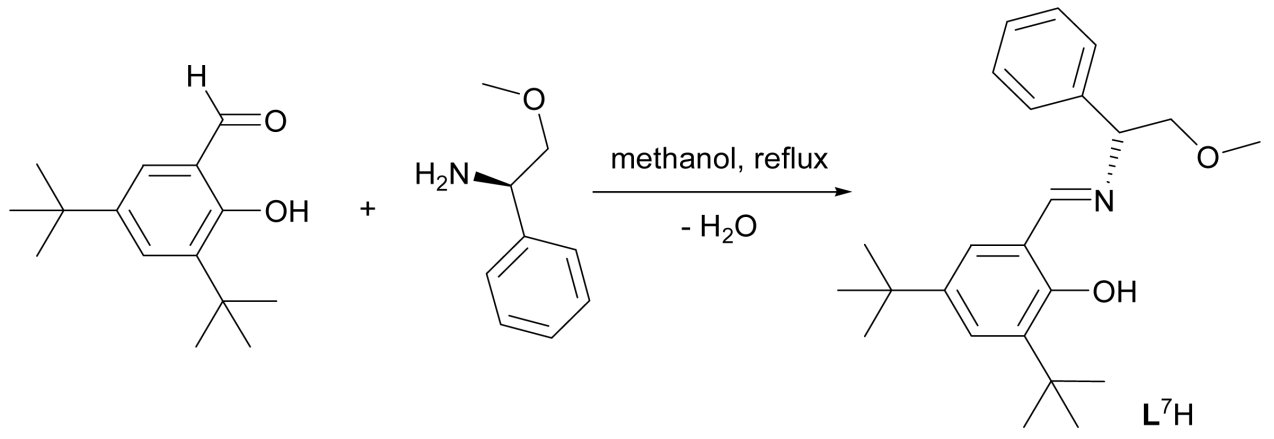

Scheme 2. Synthesis of chiral Schiff base ligand $\mathbf{L}^{7} \mathbf{H}$ via condensation reaction.

$$
\begin{aligned}
& \mathrm{Zn}\left[\mathrm{N}\left(\mathrm{SiMe}_{3}\right)_{2}\right]_{2}+\mathrm{HL} \stackrel{\text { 1. THF, }-40^{\circ} \mathrm{C}}{\text { 2. Hexanes }} \underset{\mathrm{LZn}\left[\mathrm{N}\left(\mathrm{SiMe}_{3}\right)_{2}\right]}{\longrightarrow}+\mathrm{HN}\left(\mathrm{SiMe}_{3}\right)_{2} \\
& L^{3}=3 a ; L^{4}=4 a ; L^{7}=7 a \\
& \text { HOR } \\
& \mathrm{LZn}[\mathrm{OR}]+\mathrm{HN}\left(\mathrm{SiMe}_{3}\right)_{2}
\end{aligned}
$$

Scheme 3. General synthesis of metal catalysts via ligand exchange reactions. 
d8-THF $\left(\mathrm{C}_{4} \mathrm{D}_{8} \mathrm{O}\right)$, revealed only one chemical environment consistent with a symmetrical, coordinately saturated monomeric zinc center. It is difficult to be certain of the exact nature of the asymmetry without solid state structural data. However, it was very difficult to isolate good single crystals for X-ray determination due to the extreme solubility of the zinc complexes. Meanwhile, the ${ }^{1} \mathrm{H}$ NMR spectrum of compound $7 \mathbf{a}$ demonstrated a symmetric arrangement of the ligand in either donor or non-donor solvents; indicative of a monomeric solution structure. Undoubtedly, steric constraints and additional oxygen donor atom of the [ONO] ligand imposed a monomeric zinc structure to completely fill the coordination sphere about zinc.

\subsection{X-Ray Crystal Structure of Compounds}

The results of the single crystal X-ray structure determination of ligands $\mathbf{L}^{3} \mathbf{H}$, and $\mathbf{L}^{7} \mathbf{H}$ are consistent with the formulation proposed by elemental analysis data and NMR spectroscopic analysis. Ligand $\mathbf{L}^{7} \mathbf{H}$ crystallized in the monoclinic space group $\mathrm{P} 22_{1}$ with two molecules in the asymmetric unit, while $\mathbf{L}^{3} \mathbf{H}$ crystallized in the orthorhombic space group $\mathrm{P} 2{ }_{1} 2_{1} 2_{1}$ with a single molecule in the asymmetric unit (see Figure 2 and Table 1 ). The solid state structures also confirmed the configuration of chiral centers for the ligands as indicated in Figure 1. Due to the extreme solubility of the zinc complexes in donor (THF) and non-donor solvents (hexane and toluene), it was very difficult to isolate good single crystals for X-ray determination.

\subsection{Polymerization of Lactide Using Zinc Complexes}

Compounds 3a, $\mathbf{4 a}$, and $\mathbf{7 a}$ were treated with rac-lactide under different reaction times and conditions and the results are summarized in Table 2. Except where noted, all polymerization reactions were performed solvent free at $130^{\circ} \mathrm{C}$ and quantitative conversion (based on ${ }^{1} \mathrm{H}$ NMR spectroscopy) was achieved (see Table 2). Entry 1 is the
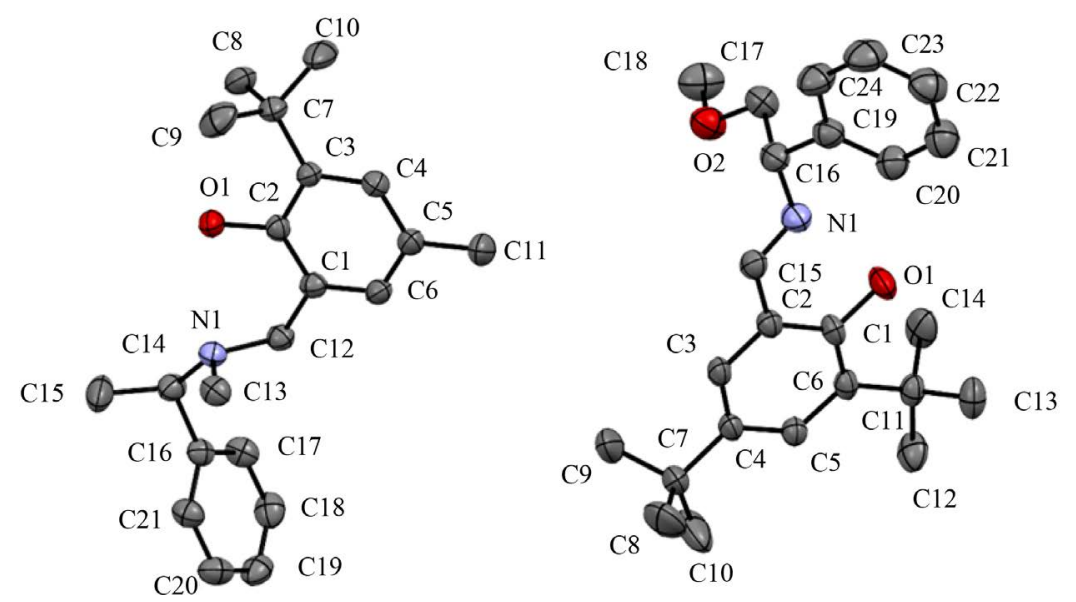

Figure 2. Molecular representations of ligands $\mathbf{L}^{3} \mathbf{H}$ and $\mathbf{L}^{7} \mathbf{H}$, respectively (hydrogen atoms have been omitted). Thermal Displacement ellipsoids are drawn at the $50 \%$ probability level.

Table 1. Crystallographic data of ligands $\mathbf{L}^{3} \mathbf{H}$ and $\mathbf{L}^{7} \mathbf{H}$.

\begin{tabular}{ccc}
\hline & $\mathbf{L}^{3} \mathbf{H}$ & $\mathbf{L}^{7} \mathbf{H}$ \\
\hline Chemical structure & $\mathrm{C}_{21} \mathrm{H}_{29} \mathrm{NO}$ & $\mathrm{C}_{24} \mathrm{H}_{33} \mathrm{NO}_{2}$ \\
Space group & $\mathrm{P} 2_{1} 2_{1} 2_{1}$ & $\mathrm{P}_{1}$ \\
Cell lengths: a, b, c $(\AA)$ & $8.8192(8), 9.1586(9), 23.020(2)$ & $10.653(6), 9.423(3), 22.096(6)$ \\
Cell volume: $\mathrm{V}\left(\AA^{3}\right)$ & 1859.36 & 2217.09 \\
$\mathrm{Z}$ & 4 & 4 \\
Cell angles: $\alpha, \beta, \gamma\left({ }^{\circ}\right)$ & $90.000,90.000,90.000$ & $90.000,91.699(13), 90.000$ \\
R-Factor $(\%)$ & 5.32 & 5.5 \\
\hline
\end{tabular}


Table 2. Polymerization of rac-Lactide using zinc complexes.

\begin{tabular}{|c|c|c|c|c|c|c|c|c|}
\hline Entry & Initiator & Co-initiator & {$[\mathrm{LA}] /[\mathrm{I}]^{\mathrm{a}}$} & Time (h) & Conv. $(\%)^{b}$ & $M_{n}\left(10^{3}\right)^{c}$ & $\mathrm{M}_{\mathrm{w}}\left(10^{3}\right)^{\mathrm{c}}$ & $\mathrm{PDI}^{\mathrm{c}}$ \\
\hline 1 & - & - & $1000^{\mathrm{d}}$ & 24 & 0 & - & - & - \\
\hline 2 & $\mathrm{~L}^{3} \mathrm{ZnN}\left(\mathrm{SiMe}_{3}\right)_{2} \mathbf{3 a}$ & - & $1000^{\mathrm{d}}$ & 1 & 0 & - & - & - \\
\hline 3 & $\mathrm{~L}^{4} \mathrm{ZnN}\left(\mathrm{SiMe}_{3}\right)_{2} \mathbf{4 a}$ & - & $1000^{\mathrm{d}}$ & 1 & 0 & - & - & - \\
\hline 4 & $\mathrm{~L}^{7} \mathrm{ZnN}\left(\mathrm{SiMe}_{3}\right)_{2} \mathbf{7 a}$ & - & $1000^{\mathrm{d}}$ & 1 & 53 & - & - & - \\
\hline 5 & $\mathrm{~L}^{3} \mathrm{ZnN}\left(\mathrm{SiMe}_{3}\right)_{2}$ 3a & - & $1000^{\mathrm{d}}$ & 24 & 100 & 7.4 & 9.9 & 1.34 \\
\hline 6 & $\mathrm{~L}^{4} \mathrm{ZnN}\left(\mathrm{SiMe}_{3}\right)_{2} \mathbf{4 a}$ & - & $1000^{\mathrm{d}}$ & 24 & 100 & 7.0 & 8.9 & 1.27 \\
\hline 7 & $\mathrm{~L}^{7} \mathrm{ZnN}\left(\mathrm{SiMe}_{3}\right)_{2} \mathbf{7 a}$ & - & $1000^{\mathrm{d}}$ & 24 & 100 & 8.9 & 11.8 & 1.29 \\
\hline 8 & $\mathrm{~L}^{7} \mathrm{ZnN}\left(\mathrm{SiMe}_{3}\right)_{2} \mathbf{7 a}$ & $\mathrm{PhCH}_{2} \mathrm{OH}$ & $100^{\mathrm{d}}$ & 24 & 100 & - & - & - \\
\hline
\end{tabular}

${ }^{a}$ Monomer to initiator ratio. ${ }^{b}$ Conversion: determined by ${ }^{1} \mathrm{H}$ NMR (integration of the methyl resonances of LA and PLA). ${ }^{\mathrm{C}} \mathrm{M}_{\mathrm{n}}$ and $\mathrm{M}_{\mathrm{w}} / \mathrm{M}_{\mathrm{n}}$ (PDI) of polymer determined by SEC with calibration to polystyrene standards. ${ }^{\mathrm{d}}$ Bulk polymerization at $130^{\circ} \mathrm{C}$.

control reaction which shows no polymerization after $24 \mathrm{~h}$. There was no reaction after 1 hour for compounds $3 a$ and 4a indicating slow initiation process presumably due to dimeric structure (Table 2, entries 2 - 3). However, the monomeric compound 7a gave 53\% conversion after 1 hour (Table 2, entry 4). All zinc complexes gave 100\% conversion after 24 hours to afford low molecular weight polylactide with narrow polydispersity (Table 2, entries 5 - 7). Compound $\left[\mathrm{L}^{7} \mathrm{ZnOCH} 2 \mathrm{Ph}\right]$ was synthesized in-situ from $7 \mathbf{a}$ and benzyl alcohol as a co-initiator in the solvent-free melt ring-opening polymerization of racemic lactide for 100 monomer-to-initiator ratio (Table 2, entry 8). NMR analysis of the polymer obtained shows the initiating group as part of the polylactide without ligand $\mathbf{L}^{7}$. All polymerization reactions were quenched with acidified methanol and polylactide was isolated as amorphous solids indicative of atactic polymer.

\subsection{Reactivity of Zinc Complexes with Lactide}

We observed moderate catalytic reactivity of rac-LA with the newly synthesized chiral zinc complexes with no stereo-control, probably due to the harsh reaction conditions with temperatures of $130^{\circ} \mathrm{C}$. No reactivity was observed with the zinc complexes when polymerization was conducted in either toluene or THF at $80^{\circ} \mathrm{C}$ after 24 hours for 1000:1 lactide-to-initiator ratio. Furthermore, increasing the amount of initiator to 100:1 under the same conditions did not yield any polymer. Due to the small size of zinc metal, in order to effectively carryout chain-growth polymerization of LA via coordination-insertion mechanism, most researchers have been successful at a temperature of $130^{\circ} \mathrm{C}$ [88]-[101]. Even though this is not somewhat surprising, it is hypothesized that attaching a larger metal such as calcium or lanthanum to the newly synthesized chiral aminophenolate ligands would provide better catalytic reactivity at milder conditions with better polymerization control.

The new chiral zinc complexes gave polymer molecular weights lower than expected due to extensive transesterification as a result of extended reaction times beyond complete polymerization reaction. It should be noted that transesterification backbiting reactions has been reported as a major cause of obtaining low molecular weight polyester chains during active polymerization process that is allowed to run over long periods without quenching the reaction [68] [70] [75]. In addition, some rac-lactide molecules were sublimed during the solvent-free bulk polymerization reactions at $130^{\circ} \mathrm{C}$ resulting in low $\mathrm{M}_{\mathrm{n}}$. Having the tert-butyl group at the ortho (3a) or para (4a) positions of the phenolate did not make any significant difference in reactivity with lactide due to small metal size.

\section{Conclusion}

We have reported new heteroleptic zinc amido complexes supported by newly synthesized chiral multidentate monoanionic amino phenolate ligands with varying steric and electronic demands. Zinc complexes were found to have moderate catalytic activity towards ring-opening polymerization of racemic lactide at $130^{\circ} \mathrm{C}$ to afford atactic polymers with molecular weight of $10,000 \mathrm{~g} / \mathrm{mol}$ and a narrow polydispersity index of 1.3. The newly synthesized chiral aminophenolate ligands are suitable chiral auxiliaries for inorganic chemists to design met- 
al-based complexes for asymmetric catalysis and stereoselective polymerization reactions.

\section{Acknowledgements}

The authors are grateful to Savannah State University's president's mini grant and US Department of Defense Army Research Office Award No. 67208-RT-REP for partial financial supports, and to Xiaolin Liu of Clemson University for help with GPC analysis.

\section{References}

[1] Wu, X.-Y., Ren, Z.-G. and Lang, J.-P. (2014) Ni(II) Tetraphosphine Complexes as Catalysts/Initiators in the Ring Opening Polymerization of $\varepsilon$-Caprolactone. Dalton Transactions, 43, 1716-1723. http://dx.doi.org/10.1039/C3DT52412D

[2] Stanlake, L.J.E., Beard, J.D. and Schafer, L.L. (2008) Rare-Earth Amidate Complexes. Easily Accessed Initiators for $\varepsilon^{-}$ Caprolactone Ring-Opening Polymerization. Inorganic Chemistry, 47, 8062-8068. http://dx.doi.org/10.1021/ic8010635

[3] Celiz, A.D. and Scherman, O.A. (2008) Controlled Ring-Opening Polymerization Initiated via Self-Complementary Hydrogen-Bonding Units. Macromolecules, 41, 4115-4119. http://dx.doi.org/10.1021/ma702699t

[4] O’Keefe, B.J., Hillmyer, M.A. and Tolman, W.B. (2001) Polymerization of Lactide and Related Cyclic Esters by Discrete Metal Complexes. Journal of the Chemical Society, Dalton Transactions, 2215-2224. http://dx.doi.org/10.1039/b104197p

[5] Coates, G.W. (2002) Polymerization Catalysis at the Millennium: Frontiers in Stereoselective, Metal-Catalyzed Polymerization. Journal of the Chemical Society, Dalton Transactions, 467-475. http://dx.doi.org/10.1039/b111226k

[6] Dechy-Cabaret, O., Martin-Vaca, B. and Bourissou, D. (2004) Controlled Ring-Opening Polymerization of Lactide and Glycolide. Chemical Reviews, 104, 6147-6176. http://dx.doi.org/10.1021/cr040002s

[7] Hou, Z. and Wakatsuki, Y. (2002) Recent Developments in Organolanthanide Polymerization Catalysts. Coordination Chemistry Reviews, 231, 1-22. http://dx.doi.org/10.1016/S0010-8545(02)00111-X

[8] Saruul, P., Sriene, F., Somers, D.A. and Samac, D.A. (2002) Production of a Biodegradable Plastic Polymer, Poly- $\beta$ Hydroxybutyrate, in Transgenic Alfalfa. Crop Science, 42, 919-927. http://dx.doi.org/10.2135/cropsci2002.0919

[9] Cameron, P.A., Gibson, V.C., Redshaw, C., Segal, J.A., White, A.J.P. and Williams, D.J. (2002) Synthesis and Characterisation of Neutral and Cationic Alkyl Aluminium Complexes Bearing N,O-Schiff Base Chelates with Pendant Donor Arms. Journal of the Chemical Society, Dalton Transactions, 415-422. http://dx.doi.org/10.1039/b106131n

[10] Tsuji, H. (2005) Poly(lactide) Stereocomplexes: Formation, Structure, Properties, Degradation, and Applications. Macromolecular Bioscience, 5, 569-597. http://dx.doi.org/10.1002/mabi.200500062

[11] Wu, J., Yu, T.-L., Chen, C.-T. and Lin, C.-C. (2006) Recent Developments in Main Group Metal Complexes Catalyzed/Initiated Polymerization of Lactides and Related Cyclic Esters. Coordination Chemistry Reviews, 250, 602-626. http://dx.doi.org/10.1016/j.ccr.2005.07.010

[12] Kricheldorf, H.R., Boettcher, C. and Tönnes, K.-U. (1992) Polylactones: 23. Polymerization of Racemic and Meso D,L-Lactide with Various Organotin Catalysts-Stereochemical Aspects. Polymer, 33, 2817-2824. http://dx.doi.org/10.1016/0032-3861(92)90459-A

[13] Chabot, F., Vert, M., Chapelle, S. and Grange, P. (1983) Configurational Structures of Lactic Acid Stereocopolymers as Determined by ${ }^{13} \mathrm{C}$ and ${ }^{1} \mathrm{H}$ nmr. Polymer, 24, 53-59. http://dx.doi.org/10.1016/0032-3861(83)90080-0

[14] Jikei, M., Takeyama, Y., Yamadoi, Y., Shinbo, N., Matsumoto, K., Motokawa, M., Ishibashi, K. and Yamamoto, F. (2015) Synthesis and Properties of Poly(L-lactide)-Poly(ع-caprolactone) Multiblock Copolymers by the Self-Polycondensation of Diblockmacromonomers. Polymer Journal, 47, 657-665. http://dx.doi.org/10.1038/pj.2015.49

[15] Haba, O. and Itabashi, H. (2014) Ring-Opening Polymerization of a Five-Membered Lactone Transfused to a Cyclohexane Ring. Polymer Journal, 46, 89-93. http://dx.doi.org/10.1038/pj.2013.70

[16] Bero, M., Kasperczyk, J. and Jedlinski, Z. (1990) Coordination Polymerization of Lactides, 1. Structure Determination of Obtained Polymers. Die Makromolekulare Chemie, 191, 2287-2296. http://dx.doi.org/10.1002/macp.1990.021911007

[17] Pltel, R.H., Hodgson, L.M. and Williams, C. (2008) Biocompatible Initiators for Lactide Polymerization. Polymer Reviews, 48, 11-63. http://dx.doi.org/10.1080/15583720701834166

[18] Joseph, J.G. (2006) Polymers for Tissue Engineering, Medical Devices, and Regenerative Medicine. Concise General Review of Recent Studies. Polymers for Advanced Technologies, 17, 395-418. http://dx.doi.org/10.1002/pat.729

[19] Fukushima, K. and Kimura, Y. (2006) Stereocomplexed Polylactides (Neo-PLA) as High-Performance Bio-Based Po- 
lymers: Their Formation, Properties, and Application. Polymer International, 55, 626-642. http://dx.doi.org/10.1002/pi.2010

[20] Albertsson, A.-C. and Varma, I.K. (2003) Recent Developments in Ring Opening Polymerization of Lactones for Biomedical Applications. Biomacromolecules, 4, 1466-1486. http://dx.doi.org/10.1021/bm034247a

[21] Ikada, Y. and Tsuji, H. (2000) Biodegradable Polyesters for Medical and Ecological Applications. Macromolecular Rapid Communications, 21, 117-132. http://dx.doi.org/10.1002/(SICI)1521-3927(20000201)21:3<117::AID-MARC117>3.0.CO;2-X

[22] Isogai, N., Asamura, S., Hagishi, T., Ikada, Y., Morita, S., Hillyer, J. and Landis, W.J. (2004) Tissue Engineering of an Auricular Cartilage Model Utilizing Cultured Chondrocyte-Poly(L-Lactide- $\varepsilon$-Caprolactone) Scaffolds. Tissue Engineering, 10, 673-687. http://dx.doi.org/10.1089/1076327041348527

[23] Benicewicz, B.C. and Hoyt, A.E. (1992) Liquid Crystal Polyester Thermosets. US Patent, US5114612 A.

[24] Daniels, A.U., Chang, M.K. and Adriano, K.P. (1990) Mechanical Properties of Biodegradable Polymers and Composites Proposed for Internal Fixation of Bone. Journal of Applied Biomaterials, 1, 57-78. http://dx.doi.org/10.1002/jab.770010109

[25] Athanasiou, K.A., Niederauer, G.G. and Agrawal, C.M. (1996) Sterilization, Toxicity, Biocompatibility, and Clinical Applications of Polylactic Acid/Polyglycolic Acid Copolymers. Biomaterials, 17, 93-102. http://dx.doi.org/10.1016/0142-9612(96)85754-1

[26] Langer, R. (1998) Drug Delivery and Targeting. Nature, 392, 5-10.

[27] Uhrich, K.E., Cannizzaro, S.M., Langer, R.S. and Shakesheff, K.M. (1999) Polymeric Systems for Controlled Drug Release. Chemical Reviews, 99, 3181-3198. http://dx.doi.org/10.1021/cr940351u

[28] Jacoby, M. (2001) Applying Engineering, Materials, and Chemistry Principles, Researchers Produce Safe, Smart, and Effective Implantable Devices. Chemical \& Engineering News, 79, 30-35. http://dx.doi.org/10.1021/cen-v079n006.p030

[29] Panyam, J. and Labhasetwar, V. (2003) Biodegradable Nanoparticles for Drug and Gene Delivery to Cells and Tissue. Advanced Drug Delivery Reviews, 55, 329-347. http://dx.doi.org/10.1016/S0169-409X(02)00228-4

[30] Mu, L. and Feng, S. (2003) A Novel Controlled Release Formulation for the Anticancer Drug Paclitaxel $\left(\right.$ Taxol $\left.^{\circledR}\right)$ : PLGA Nanoparticles Containing Vitamin E TPGS. S. Journal of Controlled Release, 86, 33-48. http://dx.doi.org/10.1016/S0168-3659(02)00320-6

[31] Kellomäki, M., Niiranen, H., Puumanen, K., Ashammakhi, N., Waris, T. and Törmälä, P. (2000) Bioabsorbable Scaffolds for Guided Bone Regeneration and Generation. Biomaterials, 21, 2495-2505. http://dx.doi.org/10.1016/S0142-9612(00)00117-4

[32] Ito, H., Minami, A., Tanino, H. and Matsuno, T. (2002) Fixation with Poly-L-lactic Acid Screws in Hip Osteotomy: 68 Hips Followed for 18 - 46 Months. Acta Orthopaedica Scandinavica, 73, 60-64. http://dx.doi.org/10.1080/000164702317281422

[33] Waris, E., Ninkovic, M., Harpf, C., Ninkovic, M. and Ashammakhi, N. (2004) Self-Reinforced Bioabsorbable Miniplates for Skeletal Fixation in Complex Hand Injury: Three Case Reports. The Journal of Hand Surgery, 29A, 452-457. http://dx.doi.org/10.1016/j.jhsa.2004.01.013

[34] Gilding, D.K. and Reed, A.M. (1979) Biodegradable Polymers for Use in Surgery-Polyglycolic Acid/Polylactic Acid Homo- and Copolymers. Polymer, 20, 1459-1464. http://dx.doi.org/10.1016/0032-3861(79)90009-0

[35] Benicewicz, B.C. and Hopper, P.K. (1990) Review: Polymers for Absorbable Surgical Sutures-Part I. Journal of Bioactive and Compatible Polymers, 5, 453-472. http://dx.doi.org/10.1177/088391159000500407

[36] Lunt, J. (1998) Large-Scale Production, Properties and Commercial Applications of Polylactic Acid Polymers. Polymer Degradation and Stability, 59, 145-152. http://dx.doi.org/10.1016/S0141-3910(97)00148-1

[37] Caldorera-Moore, M., Nicholas A. and Peppas, N.A. (2009) Micro- and Nanotechnologies for Intelligent and Responsive Biomaterial-Based Medical Systems. Advanced Drug Delivery Reviews, 16, 1391-1401. http://dx.doi.org/10.1016/j.addr.2009.09.002

[38] Slager, J. and Domb, A.J. (2003) Biopolymer Stereocomplexes. Advanced Drug Delivery Reviews, 55, 549-583. http://dx.doi.org/10.1016/S0169-409X(03)00042-5

[39] Gollwitzer, H., Ibrahim, K., Meyer, H., Mittelmeier, W., Busch, R. and Stemberger, A. (2003) Antibacterial Poly(D,Llactic Acid) Coating of Medical Implants Using a Biodegradable Drug Delivery Technology. Journal of Antimicrobial Chemotherapy, 51, 585-591. http://dx.doi.org/10.1093/jac/dkg105

[40] Lendlein, A. and Kelch, S. (2002) Shape-Memory Polymers. Angewandte Chemie International Edition, 41, $2034-2057$. http://dx.doi.org/10.1002/1521-3773(20020617)41:12<2034::AID-ANIE2034>3.0.CO;2-M

[41] Lendlein, A. and Langer, R. (2002) Biodegradable, Elastic Shape-Memory Polymers for Potential Biomedical Applica- 
tions. Science, 296, 1673-1676. http://dx.doi.org/10.1126/science.1066102

[42] Aoyagi, T., Miyata, F. and Nagase, Y. (1994) Preparation of Cross-Linked Aliphatic Polyester and Application to Thermos-Responsive Materials. Journal of Controlled Release, 32, 87-96. http://dx.doi.org/10.1016/0168-3659(94)90228-3

[43] Radano, C.P., Baker, G.L. and Smith, M.R. (2000) Stereoselective Polymerization of a Racemic Monomer with a Racemic Catalyst: Direct Preparation of the Polylactic Acid Stereocomplex from Racemic Lactide. Journal of the American Chemical Society, 122, 1552-1553. http://dx.doi.org/10.1021/ja9930519

[44] Darensbourg, D.J., Choi, W., Karroonnirum, O. and Bhuvanesh, N. (2008) Ring-Opening Polymerization of Cyclic Monomers by Complexes Derived from Biocompatible Metals. Production of Poly(Lactide), Poly(Trimethylene Carbonate), and Their Copolymers. Macromolecules, 41, 3493-3502. http://dx.doi.org/10.1021/ma800078t

[45] Ovitt, T.M. and Coates, G.W. (2002) Stereochemistry of Lactide Polymerization with Chiral Catalysts: New Opportunities for Stereocontrol Using Polymer Exchange Mechanisms. Journal of the American Chemical Society, 124, 1316-1326. http://dx.doi.org/10.1021/ja012052+

[46] Kasperczyk, J.E. (1995) Microstructure Analysis of Poly(Lactic Acid) Obtained by Lithium Tert-Butoxide as Initiator. Macromolecules, 28, 3937-3939. http://pubs.acs.org/doi/abs/10.1021/ma00115a028 http://dx.doi.org/10.1021/ma00115a028

[47] Rajashekhar, B., Roymuhury, S.K., Chakraborty, D. and Ramkumar, V. (2015) Group 4 Metal Complexes of Trost’s Semi-Crown Ligand: Synthesis, Structural Characterization and Studies on the Ring-Opening Polymerization of Lactides and $\varepsilon$-Caprolactone. Dalton Transactions, 44, 16280-16293. http://dx.doi.org/10.1039/C5DT02267C

[48] Thakur, K.A.M., Kean, R.T., Zell, M.T., Padden, B.E. and Munson, E.J. (1998) An Alternative Interpretation of the HETCOR NMR Spectra of Poly(Lactide). Chemical Communications, No. 17, 1913-1914. http://dx.doi.org/10.1039/a708911b

[49] Thakur, K.A.M., Kean, R.T., Hall, E.S., Kolstad, J.J. and Lindgren, T.A. (1997) High-Resolution ${ }^{13} \mathrm{C}$ and ${ }^{1}$ H Solution NMR Study of Poly(Lactide). Macromolecules, 30, 2422-2428. http://dx.doi.org/10.1021/ma9615967

[50] Zell, M.T., Padden, B.E., Paterick, A.J., Thakur, K.A.M., Kean, R.T., Hillmyer, M.A. and Munson, E.J. (2002) Unambiguous Determination of the ${ }^{13} \mathrm{C}$ and ${ }^{1} \mathrm{H}$ NMR Stereosequence Assignments of Polylactide Using High-Resolution Solution NMR Spectroscopy. Macromolecules, 35, 7700-7707. http://dx.doi.org/10.1021/ma0204148

[51] Ovitt, T.M. and Coates, G.W. (2000) Stereoselective Ring-Opening Polymerization of rac-Lactide with a Single-Site, Racemic Aluminum Alkoxide Catalyst: Synthesis of Stereoblockpoly(Lactic Acid). Polymer Chemistry, 38, 4686-4692. http://dx.doi.org/10.1002/1099-0518(200012)38:1+<4686::AID-POLA80>3.0.CO;2-0

[52] Cheng, M., Attygalle, A.B., Lobkovsky, E.B. and Coates, G.W. (1999) Single-Site Catalysts for Ring-Opening Polymerization: Synthesis of Heterotactic Poly(Lactic Acid) from rac-Lactide. Journal of the American Chemical Society, 121, 11583-11584. http://dx.doi.org/10.1021/ja9926780

[53] Ovitt, T.M. and Coates, G.W. (1999) Stereoselective Ring-Opening Polymerization of meso-Lactide: Synthesis of Syndiotactic Poly(Lactic Acid). Journal of the American Chemical Society, 121, 4072-4073. http://dx.doi.org/10.1021/ja990088k

[54] Chisholm, M.H., Iyer, S.S., McCollum, D.G., Pagel, M. and Werner-Zwanziger, U. (1999) Microstructure of Poly (Lactide). Phase-Sensitive HETCOR Spectra of Poly(meso-Lactide), Poly(rac-Lactide), and Atactic Poly(Lactide). Macromolecules, 32, 963-973. http://dx.doi.org/10.1021/ma9806864

[55] Chisholm, M.H., Gallucci, J.C. and Phomphrai, K. (2005) Comparative Study of the Coordination Chemistry and Lactide Polymerization of Alkoxide and Amide Complexes of Zinc and Magnesium with a $\beta$-Diiminato Ligand Bearing Ether Substituents. Inorganic Chemistry, 44, 8004-8010. http://dx.doi.org/10.1021/ic048363d

[56] Zhong, Z., Dijkstra, P.J. and Feijen, J. (2003) Controlled and Stereoselective Polymerization of Lactide: Kinetics, Selectivity, and Microstructures. Journal of the American Chemical Society, 125, 11291-11298. http://dx.doi.org/10.1021/ja0347585

[57] Cai, C.-X., Amgoune, A., Lehmann, C.W. and Carpentier, J.-F. (2004) Stereoselective Ring-Opening Polymerization of Racemic Lactide Using Alkoxy-Amino-Bis(Phenolate) Group 3 Metal Complexes. Chemical Communications, No. 3, 330-331. http://dx.doi.org/10.1039/b314030j

[58] Coates, G.W. (2000) Precise Control of Polyolefin Stereochemistry Using Single-Site Metal Catalysts. Chemical Reviews, 100, 1223-1252. http://dx.doi.org/10.1021/cr990286u

[59] Nimitsiriwat, N., Marshall, E.L., Gibson, V.C., Elsegood, M.R.J. and Dale, S.H. (2004) Unprecedented Reversible Migration of Amide to Schiff Base Ligands Attached to Tin: Latent Single-Site Initiators for Lactide Polymerization. Journal of the American Chemical Society, 126, 13598-13599. http://dx.doi.org/10.1021/ja0470315

[60] Chisholm, M.H., Eilerts, N.W., Huffman, J.C., Iyer, S.S., Pacold, M. and Phomphrai, K. (2000) Molecular Design of Single-Site Metal Alkoxide Catalyst Precursors for Ring-Opening Polymerization Reactions Leading to Polyoxyge- 
nates. 1. Polylactide Formation by Achiral and Chiral Magnesium and Zinc Alkoxides, $\left(\eta^{3}-\mathrm{L}\right) \mathrm{MOR}$, Where L $=$ Trispyrazolyl- and Trisindazolylborate Ligands. Journal of the American Chemical Society, 122, 11845-11854. http://dx.doi.org/10.1021/ja002160g

[61] Cai, C.X., Toupet, L., Lehmann, C.W. and Carpentier, J.-F. (2003) Synthesis, Structure and Reactivity of New Yttrium Bis(Dimethylsilyl)Amido and Bis(Trimethylsilyl)Methyl Complexes of a Tetradentatebis(Phenoxide) Ligand. Journal of Organometallic Chemistry, 683, 131-136. http://dx.doi.org/10.1016/S0022-328X(03)00513-8

[62] Chamberlain, B.M., Cheng, M., Moore, D.R., Ovitt, T.M., Lobkovsky, E.B. and Coates, G.W. (2001) Polymerization of Lactide with Zinc and Magnesium $\beta$-Diiminate Complexes: Stereocontrol and Mechanism. Journal of the American Chemical Society, 123, 3229-3238. http://dx.doi.org/10.1021/ja003851f

[63] Kowalski, A., Duda, A. and Penczek, S. (1998) Polymerization of L,L-Lactide Initiated by Aluminum Isopropoxide Trimer or Tetramer. Macromolecules, 31, 2114-2122. http://dx.doi.org/10.1021/ma971737k

[64] Dove, A.P., Gibson, V.C., Marshall, E.L., White, A.J.P. and Williams, D. (2001) A Well Defined Tin(II) Initiator for the Living Polymerisation of Lactide. Chemical Communications, No. 3, 283-284. http://dx.doi.org/10.1039/b008770j

[65] Grunova, E., Kirillov, E., Roisnel, T. and Carpentier, J.-F. (2008) Group 3 Metal Complexes of Salen-like FluorousDialkoxy-Diimino Ligands: Synthesis, Structure, and Application in Ring-Opening Polymerization of rac-Lactide and rac- $\beta$-Butyrolactone.Organometallics, 27, 5691-5698. http://dx.doi.org/10.1021/om800611c

[66] Binda, P.I., Delbridge, E.E., Dugah, D.T., Skelton, B.W. and White, A.H. (2008) Synthesis and Structural Characterization of Some Potassium Complexes of Some Bis(Phenolate) Ligands and Some Novel Heterobimetallic Binuclear Arrays Formed with Trivalent Lanthanoid Ions. Zeitschrift für Anorganische und Allgemeine Chemie, 634, 325-334. http://dx.doi.org/10.1002/zaac.200700442

[67] Delbridge, E.E., Dugah, D.T., Nelson, C.R., Skelton, B.W. and White, A.H. (2007) Synthesis, Structure and Oxidation of New Ytterbium(II) Bis(Phenolate) Compounds and Their Catalytic Activity towards $\varepsilon$-Caprolactone. Dalton Transactions, No. 1, 143-153. http://dx.doi.org/10.1039/B613409B

[68] Binda, P.I. and Delbridge, E.E. (2007) Synthesis and Characterisation of Lanthanide Phenolate Compounds and Their Catalytic Activity towards Ring-Opening Polymerisation of Cyclic Esters. Dalton Transactions, No. 41, 4685-4692. http://dx.doi.org/10.1039/b710070a

[69] Kerton, F.M., Whitwood, A.C. and Willans, C.E. (2004) A High-Throughput Approach to Lanthanide Complexes and Their Rapid Screening in the Ring Opening Polymerisation of Caprolactone. Dalton Transactions, No. 15, $2237-2244$. http://dx.doi.org/10.1039/b406841f

[70] Binda, P.I., Delbridge, E.E., Abrahamson, H.B. and Skelton, B.W. (2009) Coordination of Substitutionally Inert Phenolate Ligands to Lanthanide(II) and (III) Compounds-Catalysts for Ring-Opening Polymerization of Cyclic Esters. Dalton Transactions, No. 15, 2777-2787. http://dx.doi.org/10.1039/b821770j

[71] Miura, M. (2004) Rational Ligand Design in Constructing Efficient Catalyst Systems for Suzuki-Miyaura Coupling. Angewandte Chemie International Edition, 43, 2201-2203. http://dx.doi.org/10.1002/anie.200301753

[72] Katz, B.A., Cass, R.T., Liu, B., Arze, R. and Collins, N. (2005) Functional Characterization of Novel Human and Mouse Equilibrative Nucleoside Transporters (hENT3 and mENT3) Located in Intracellular Membranes. The Journal of Biological Chemistry, 270, 31210-31218.

[73] Falciola, C.A. and Alexakis, A. (2008) Copper-Catalyzed Asymmetric Allylic Alkylation. European Journal of Organic Chemistry, No. 2008, 3765-3780. http://dx.doi.org/10.1002/ejoc.200800025

[74] Heitbaum, M., Glorius, F. and Escher, I. (2006) Asymmetric Heterogeneous Catalysis. Angewandte Chemie International Edition, 45, 4732-4762. http://dx.doi.org/10.1002/anie.200504212

[75] Dugah, D.T., Skelton, B.W. and Delbridge, E.E. (2009) Synthesis and Characterization of New Divalent Lanthanide Complexes Supported by Amine Bis(Phenolate) Ligands and Their Applications in the Ring Opening Polymerization of Cyclic Esters. Dalton Transactions, No. 8, 1436-1445. http://dx.doi.org/10.1039/b816916k

[76] Xu, X., Ma, M., Yao, Y., Zhang, Y. and Shen, Q. (2005) Synthesis, Characterisation of Carbon-Bridged (Diphenolato)Lanthanide Complexes and Their Catalytic Activity for Diels-Alder Reactions. European Journal of Inorganic Chemistry, 2005, 676-684. http://dx.doi.org/10.1002/ejic.200400519

[77] Lendlein, A., Schmidt, A.M., Schroeter, M. and Langer, R. (2005) Shape-Memory Polymer Networks from Oligo(€Caprolactone)Dimethacrylates. Journal of Polymer Science Part A: Polymer Chemistry, 43, 1369-1381. http://dx.doi.org/10.1002/pola.20598

[78] Amgoune, A., Thomas, C.M., Roisnel, T. and Carpentier, J.F. (2005) Ring-Opening Polymerization of Lactide with Group 3 Metal Complexes Supported by Dianionic Alkoxy-Amino-Bisphenolate Ligands: Combining High Activity, Productivity, and Selectivity. Chemistry-A European Journal, 12, 169-179. http://dx.doi.org/10.1002/chem.200500856

[79] Guo, H., Zhou, H., Yao, Y., Zhang, Y. and Shen, Q. (2007) Synthesis and Structural Characterization of Novel Mixed- 
Valent Samarium and Divalent Ytterbium and Europium Complexes Supported by Amine Bis(Phenolate) Ligands. Dalton Transactions, No. 32, 3555-3561. http://dx.doi.org/10.1039/b705353c

[80] Zats, G.M., Arora, H., Lavi, R., Yufit, D. and Benisvy, L. (2011) Phenolate and Phenoxyl Radical Complexes of Cu(II) and Co(III), Bearing a New Redox Active N,O-Phenol-Pyrazole Ligand. Dalton Transactions, 40, 10889-10896. http://dx.doi.org/10.1039/c1dt10615e

[81] Li, W., Xue, M., Tu, J., Zhang, Y. and Shen, Q. (2012) Syntheses and Structures of Lanthanide Borohydrides Supported by a Bridged Bis(Amidinate) Ligand and Their High Activity for Controlled Polymerization of $\varepsilon$-Caprolactone, L-Lactide and rac-Lactide. Dalton Transactions, 41, 7258-7265. http://dx.doi.org/10.1039/c2dt30096f

[82] Chisholm, M.H. and Zhou, Z. (2004) New Generation Polymers: The Role of Metal Alkoxides as Catalysts in the Production of Polyoxygenates. Journal of Materials Chemistry, 14, 3081-3092. http://dx.doi.org/10.1039/b405489j

[83] Darensburg, D.J. and Karroonnirun, O. (2010) Ring-Opening Polymerization of Lactides Catalyzed by Natural Amino-Acid Based Zinc Catalysts. Inorganic Chemistry, 49, 2360-2371. http://dx.doi.org/10.1021/ic902271x

[84] Tsuji, H. and Ikada, Y. (1999) Stereocomplex Formation between Enantiomeric Poly(Lactic Acid)s. XI. Mechanical Properties and Morphology of Solution-Cast Films. Polymer, 40, 6699-6708. http://dx.doi.org/10.1016/S0032-3861(99)00004-X

[85] Spinu, M., Jackson, C. and Keating, M.Y. (1996) Stereoselective Polymerization with Single-Site Catalysts. Journal of Macromolecular Science, Part A: Pure and Applied Chemistry, A33, 1497-1530. http://dx.doi.org/10.1080/10601329608014922

[86] Sarasua, J.-R., Prud'homme, R.E., Wisniewski, M., Le Borgne, A. and Spassky, N. (1998) Crystallization and Melting Behavior of Polylac Tides. Macromolecules, 31, 3895-3905. http://dx.doi.org/10.1021/ma971545p

[87] Kobayashi, K., Sumitomo, H. and Itoigawa, T. (1987) Maltopentaose- and Maltoheptaose-Carrying Styrene Macromers and Their Homopolymers. Macromolecules, 20, 906-908. http://dx.doi.org/10.1021/ma00170a035

[88] Wheaton, C.A., Hayes, P.G. and Ireland, B.J. (2009) Complexes of Mg, Ca and Zn as Homogeneous Catalysts for Lactide Polymerization. Dalton Transactions, No. 25, 4832-4846. http://dx.doi.org/10.1039/b819107g

[89] Wang, L. and Ma, H. (2010) Zinc Complexes Supported by Multidentate Aminophenolate Ligands: Synthesis, Structure and Catalysis in Ring-Opening Polymerization of rac-Lactide. Dalton Transactions, 39, 7897-7910. http://dx.doi.org/10.1039/c0dt00250j

[90] Song, S., Zhang, X., Ma, H. and Yang, Y. (2012) Zinc Complexes Supported by Claw-Type Aminophenolate Ligands: Synthesis, Characterization and Catalysis in the Ring-Opening Polymerization of rac-Lactide. Dalton Transactions, 41, 3266-3277. http://dx.doi.org/10.1039/c2dt11767c

[91] Drouin, F., Oguadinma, P.O., Whitehorne, T.J.J., Prud'homme, R.E. and Schaper, F. (2010) Lactide Polymerization with Chiral $\beta$-Diketiminate Zinc Complexes. Organometallics, 29, 2139-2147. http://dx.doi.org/10.1021/om100154w

[92] Labourdette, G., Lee, D.J., Patrick, B.O., Ezhova, M.B. and Mehrkhodavandi, P. (2009) Unusually Stable Chiral Ethyl Zinc Complexes: Reactivity and Polymerization of Lactide. Organometallics, 28, 1309-1319. http://dx.doi.org/10.1021/om800818v

[93] Börner, J., Vieira, I.S., Pawlis, A., Döring, A., Kuckling, D. and Pawlis, S.H. (2011) Mechanism of the Living Lactide Polymerization Mediated by Robust Zinc Guanidine Complexes. Chemistry—A European Journal, 17, 4507-4512. http://dx.doi.org/10.1002/chem.201002690

[94] Börner, J., Flöke, U., Huber, K., Döring, A., Kuckling, D. and Pawlis, S.H. (2009) Lactide Polymerisation with AirStable and Highly Active Zinc Complexes with Guanidine-Pyridine Hybrid Ligands. Chemistry-A European Journal, 15, 2362-2376. http://dx.doi.org/10.1002/chem.200802128

[95] Garcés, A., Sánchez-Barba, L.F., Alonso-Moreno, C., Fajardo, M., Fernández-Baeza, J., Otero, A., Lara-Sánchez, A., López-Solera, I. and Rodriguez, A.M. (2010) Hybrid Scorpionate/Cyclopentadienyl Magnesium and Zinc Complexes: Synthesis, Coordination Chemistry, and Ring-Opening Polymerization Studies on Cyclic Esters. Inorganic Chemistry, 49, 2859-2871. http://dx.doi.org/10.1021/ic902399r

[96] Gao, B., Duan, R., Pang, X., Li, X., Qu, Z., Shao, H., Wang, X. and Chen, X. (2013) Zinc Complexes Containing Asymmetrical N,N,O-Tridentate Ligands and Their Application in Lactide Polymerization. Dalton Transactions, 42, 16334-16342. http://dx.doi.org/10.1039/c3dt52016a

[97] Tschan, M.J.-T., Guo, J., Raman, S.K., Brulé, E., Roisnel, T., Marie-Noelle Rager, M.-N., Legay, R., Durieux, G., Rigaud, B. and Thomas, C.M. (2014) Zinc and Cobalt Complexes Based on Tripodal Ligands: Synthesis, Structure and Reactivity toward Lactide. Dalton Transactions, 43, 4550-4564. http://dx.doi.org/10.1039/c3dt52629a

[98] Hens, A., Mondal, P. and Rajak, K.K. (2013) Synthesis, Structure and Spectral Properties of $O, N, N$ Coordinating Ligands and Their Neutral Zn(II) Complexes: A Combined Experimental and Theoretical Study. Dalton Transactions, 43, 14905-14915. http://dx.doi.org/10.1039/c3dt51571k

[99] Ikpo, N., Saunders, L.N., Walsh, J.L., Smith, J.M.B., Dawe, L.N. and Kerton, F.M. (2011) Zinc Complexes of Pipera- 
zinyl-Derived Aminephenolate Ligands: Synthesis, Characterization and Ring-Opening Polymerization Activity. European Journal of Inorganic Chemistry, 2011, 5347-5359. http://dx.doi.org/10.1002/ejic.201100703

[100] Mou, Z., Liu, B., Wang, M., Xie, H., Li, P., Li, L., Li, S. and Cui, D. (2014) Isoselective Ring-Opening Polymerization of rac-Lactide Initiated by Achiral Heteroscorpionate Zwitterionic Zinc Complexes. Chemical Communications, 50, 11411-11414. http://dx.doi.org/10.1039/C4CC05033A

[101] Wang, H. and Ma, H. (2013) Highly Diastereoselective Synthesis of Chiral Aminophenolate Zinc Complexes and Isoselective Polymerization of rac-Lactide. Chemical Communications, 49, 8686-8688. http://dx.doi.org/10.1039/c3cc44980g

\section{Submit or recommend next manuscript to SCIRP and we will provide best service for you:}

Accepting pre-submission inquiries through Email, Facebook, LinkedIn, Twitter, etc.

A wide selection of journals (inclusive of 9 subjects, more than 200 journals)

Providing 24-hour high-quality service

User-friendly online submission system

Fair and swift peer-review system

Efficient typesetting and proofreading procedure

Display of the result of downloads and visits, as well as the number of cited articles

Maximum dissemination of your research work

Submit your manuscript at: http://papersubmission.scirp.org/ 